\title{
Power Control for Cognitive Radio Networks under Imperfect CSI Based on Game Theory
}

\author{
Guanglong Yang ${ }^{1,2}$, Xiao Wang ${ }^{1,2}$ and Xuezhi Tan ${ }^{1,2}$ \\ ${ }^{1}$ Communication Research Center, Harbin Institute of Technology, Harbin, 150080, \\ China \\ ${ }^{2}$ Key Laboratory of Police Wireless Digital Communication, Ministry of Public \\ Security, Harbin, 150080, China. \\ E-mail:hit12b@126.com; hitwx@hit.edu.cn;tanxz1957@hit.edu.cn
}

\begin{abstract}
In this paper, the problem of power control for cognitive systems is studied via game theory. The objective is to maximize the sum utility of secondary users (SUs) subject to the primary users (PUS) interference constraints, the transmission power constraints of SUs, and the signal-to-interference-plus-noise ratio (SINR) constraint of each SU. In our earlier work, the problem was formulated as a non-cooperative game under the assumption of perfect channel state information (CSI). Nash equilibrium (NE) is considered as the solution of this game. A distributed algorithm is proposed which can converge to the NE. Due to the limited cooperation between the secondary base station (SBS) and the PU, imperfect CSI between the $S B S$ and the PU is further considered in this work. The problem is formulated as a robust game. As it is difficult to solve the optimization problem in this case, existence of the NE cannot be analyzed. Therefore, convergence property of the sum utility of SUs will be illustrated numerically. Simulation results show that under imperfect CSI the proposed approach can converge to a locally optimal transmission power level, while the sum utility of SUs converges with the increase of the transmission power constraint of SUs.
\end{abstract}

Keywords: Cognitive radio, power control, imperfect channel state information, game theory.

\section{Introduction}

With a dramatic increasing in wireless communication applications, the shortage of spectrum resources becomes more and more serious. In order to improve the low efficiency of spectrum utilization, cognitive radio (CR) is a new promising technology to solve the problem of spectral scarcity and underutilization due to the fixed spectrum allocation methods. In CR networks, through a flexible spectrum access technology, secondary users (SUs) are allowed to identify and access the available frequency bands, which are not temporarily used by primary users (PUs) as long as the total interference power at PU's receiver is kept below a certain threshold called the interference temperature level [1].

Power control is a key technology to solve the radio resource allocation problem in CR networks, which must meet different communication qualities through adaptively regulating the system parameters and guaranteeing the interference power from SUs to PUs below the given threshold. In this area, several results have been obtained under the assumption of perfect channel state information (CSI). In [2], a novel Nash equilibrium problem based on variational inequality theory is proposed to solve the resource allocation in CR system with the objective to maximize sum rate, but the SINR requirement of SU is not considered. In [3], 
a power consumption minimization problem is formulated under a sum rate constraint for a cognitive MIMO system. In [4, 5], power control problems with the objective of utility maximization are investigated to provide reliable transmission opportunities for SUs. Based on game theory, optimal power control algorithms are proposed for CR networks in [6, 7]. With the assumption of knowing the exact CSI, the optimal solutions for resource allocation problems are proposed for different system models in [8,9]. Although in the above cited references, they have generally assumed that the perfect CSI are available to SUs. However, it is impossible for SUs to exactly obtain the complete system information due to feedback delays and quantization errors. In addition, lack of the consideration of uncertain parameters may cause undesired results in practical system.

Uncertainty in game theory has only recently been investigated. In [10], following the worst-case robust theory, the authors took into account the imperfectness of SU-to-PU CSI by adopting proper interference constraints. The existence and uniqueness of the NE of the robust game is studied by relying on the variational inequality theory. A distribution-free robust framework for the rate-maximization game has been proposed in [11], where the authors analyzed the social properties of the equilibrium under varying channel uncertainty bounds for the two-user case. Robust equilibrium in additively coupled games in communications networks has been presented in [12]. The objective is to present a complete analysis of the $\mathrm{NE}$ in robust games as compared to that of $\mathrm{NE}$ in nominal games with complete information.

Motivated by the mentioned above works, in this paper, we address the optimal power control strategies for SU to maximize the sum utilities under imperfect CSI via game theory. Besides the interference power constraint to protect PUs, we also consider the transmission power constraint of SUs and the SINR constraints of each SU. Compared with the existing works, it is cumbersome to find the optimal solution power allocation through convex optimization because the objective function in the optimization problem is non-convex. Therefore, a non-cooperative game is formulated and the NE is considered as the solution of this game. However, due to the lack of cooperation between SBS and PUs in practice, in this work the imperfect CSI is taken into account by the robust interference constraint. The optimization problem in the formulated robust game is converted into a (second order cone programming) SOCP problem.

The rest of this paper is organized as follows. The CR system model is introduced in Section 2. The game formulated under imperfect CSI is discussed in Section 3. Simulation results are presented in Section 4. Some concluding remarks are made in Section 5.

\section{System Model and Problem Formulation}

As shown in Figure 1 we consider a coexisting system where a primary network consisting of a primary base station (PBS) and $M$ PUs coexists with a secondary network with a SBS and $K$ SUs. In the secondary network, SUs operate in the frequency band allocated to the PUs, thus the channels between the base stations and users are inherently interference channels. We consider the downlink of this cognitive network in which the SBS has $N_{t}$ antennas and each SU has only one antenna, while both the PBS and the PU are equipped with a single antenna. For simplicity, we assume a flat fading channel model. Let $h_{k}$, $k \in[1, K]$, and $g_{m}, \quad m \in[1, M]$ denote the channels from the SBS to $S U_{k}$ and to $P U_{m}$, 
respectively. Similarly, channels between PBS and the $P U_{m}$ and between PBS and $S U_{k}$ are denoted by $\hat{h}_{m}$ and $\hat{g}_{k}$, respectively. Then the received signal at $S U_{k}$ is represented as

$$
y_{k}=\sqrt{p_{k}} h_{k} s_{k}+h_{k} \sum_{i=1, i \neq k}^{k} \sqrt{p_{i}} s_{i}+\hat{g}_{k} \sum_{m=1}^{M} \sqrt{p_{m}} s_{m}+n_{k}
$$

where $s_{k}$ and $s_{m}$ are the transmitted signals from SBS to $S U_{k}$ and that from PBS to $P U_{m}$, respectively. Likewise, $p_{k}$ and $p_{m}$ account for the transmission power of $S U_{k}$ and $P U_{m}$, respectively. And $n_{k}$ is an additive white Gaussian noise with zero mean and variance $\sigma_{k}^{2}$.

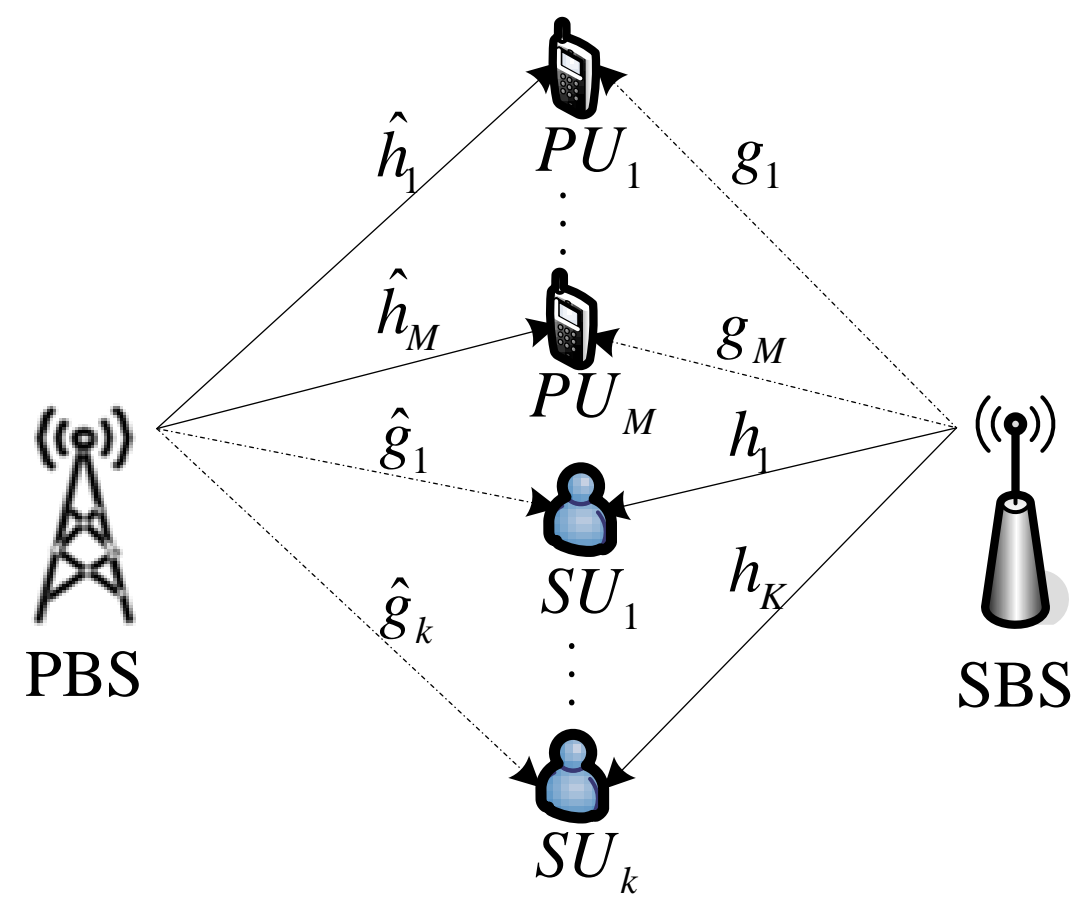

Figure 1. Cognitive Radio System Model Solid line denotes Transmission Channel and Dotted Line Denotes Interference Channel

Since signals are transmitted to multiple SUs in the same frequency band, signals transmitted to a particular SU could interfere with other secondary users. The SINR for the $S U_{k}$ can be written as

$$
\operatorname{SINR}_{k}=\frac{\left|h_{k}\right|^{2} p_{k}}{\sum_{i=1, i \neq k}^{K}\left|h_{k}\right|^{2} p_{i}+\sum_{j=1}^{M}\left|\hat{g}_{k}\right|^{2} p_{m}+\sigma_{k}^{2}}
$$

Since SUs and PUs could share the same frequency band, communication between SUs introduces interference to PUs. The interference power $p_{m, t h}$ at the $P U_{m}$ due to the SU transmission can be given by [2]

$$
\sum_{k=1}^{K}\left|g_{m}\right|^{2} p_{k} \leq p_{m, t h}
$$


To guarantee the QoS requirements of SUs, the SINR at each SU should be larger than a threshold $\gamma_{k, t h}$ [13], i.e., we have

$$
\operatorname{SIN} R_{k} \geq \gamma_{k, t h}
$$

In practice, the CSI between SBS and PUs is often imperfect due to lack of cooperation, but each SU knows the interference threshold value. Under ellipsoid approximation [10], the true channel coefficient $g_{m}$ can be written as

$$
g_{m}=\tilde{g}_{m}+\Delta_{m}
$$

where $\tilde{g}_{m}$ is the estimated value of channel gain at the SBS and $\Delta_{m}$ is a norm-bounded uncertain vector, namely,

$$
\left|\Delta_{m}\right|^{2} \leq c_{m} \varepsilon_{m}^{2}
$$

Here, $c_{m}$ and $\varepsilon_{m}^{2}$ are uncertainty parameter and estimation error, respectively.

The achievable rate at $S U_{k}$ writes

$$
\begin{aligned}
& R_{k}=\log \left(1+\operatorname{SIN} R_{k}\right) \\
& =\log \left(1+\frac{\left|h_{k}\right|^{2} p_{k}}{\sum_{i=1, i \neq k}^{K}\left|h_{k}\right|^{2} p_{i}+\sum_{j=1}^{M}\left|\hat{g}_{k}\right|^{2} p_{m}+\sigma_{k}^{2}}\right)
\end{aligned}
$$

Where $\sum_{j=1}^{M}\left|\hat{g}_{k}\right|^{2} p_{m}$ is the interference introduced by PUs.

To improve the system performance of the secondary network, we employ a power control strategy to maximize its sum rate under the interference constraints at PUs, the total transmission power constraint of SUs, and the target SINRs for each SU. The optimization problem can be stated as,

$$
\begin{aligned}
\underset{p_{k}}{\max } & \sum_{k=1}^{K} R_{k} \\
& \sum_{k=1}^{K}\left|g_{m}\right|^{2} p_{k} \leq p_{m, t h} \\
\text { s.t. } & \sum_{k=1}^{K} p_{k} \leq P_{T} \\
& S I N R_{k} \geq \gamma_{k, t h}
\end{aligned}
$$

So, the robust power control problem that considers the channel uncertainty can be written as

$$
\max _{p_{k}} \sum_{k=1}^{K} R_{k}
$$

$$
\begin{aligned}
& \sum_{k=1}^{K}\left|\hat{g}_{m}+\Delta_{m}\right|^{2} p_{k} \leq p_{m, t h} \\
\text { s.t. } & \sum_{k=1}^{K} p_{k} \leq P_{T} \\
& \operatorname{SIN} R_{k} \geq \gamma_{k, t h} \\
& \left|\Delta_{m}\right|^{2} \leq c_{m} \varepsilon_{m}^{2}
\end{aligned}
$$




\section{Power Control Game under Imperfect CSI}

\section{A. Game-theoretic Formulation}

Game theory is an effective tool to deal with the strategy choice and balancing among individuals who are of conflict interests. The participants in a game with conflict interests will selfishly choose their own strategies to maximize their utility functions. In CR system, if a SU selfishly raises transmission power to increase its own utility, this will inevitably increase the interference to other SUs, thus resulting in mutual interference between SUs [7]. To solve the problem, a balance point should be found when SUs use channel to transmit data, so the power control problem can be abstracted as a non-cooperative power control game from the perspective of game theory. Based on the system model described above, the non-cooperative game can be formulated as follows

$$
G=\left[N,\left\{\mathbf{P}_{i}\right\},\left\{u_{i}(\cdot)\right\}\right]
$$

It has the following components:

- Players: in this paper is SUs, it can be denoted: $i=\{1,2,3, \cdots, N\}$.

- Strategic Space: it is defined by the transmission power allocation strategy. For each available power level $p_{i} \in \mathrm{s}_{i}$, the strategy space is defined as: $\mathrm{S}=p_{1} \times p_{2} \times \cdots \times p_{N}$.

- Utility Function: the utility function of $\mathrm{SU} i$ is denoted as $U_{i}\left(p_{i}, \mathrm{p}_{-i}\right)$, where $\mathrm{p}_{-i}$ is the power vector of all SUs except $S U_{i}$.

Under the assumption of imperfect CSI between the SBS and PUs, in order to enable the SUs to share the spectrum with the PU, we should find appropriate power weights to distribute them among the SUs so that the sum utility of SUs is maximized and the interference created to the PU is as low as possible. This can be described by a robust game, i.e., the SUs compete with each other to maximize the sum utility under imperfect CSI.

Adopting the worst-case CSI uncertainty model [10], the robust game $G^{\text {rob }}$ can be formulated as

$$
\begin{aligned}
\max _{p_{k}} & \sum_{k=1}^{K} R_{k}^{r o b} \\
& \sum_{k=1}^{K}\left|\tilde{g}_{m}+\Delta_{m}\right|^{2} p_{k} \leq p_{m, t h} \\
\text { s.t. } & \sum_{k=1}^{K} p_{k} \leq P_{T} \\
& S I N R_{k} \geq \gamma_{k, t h} \\
& \left|\Delta_{m}\right|^{2} \leq c_{m} \varepsilon_{m}^{2}
\end{aligned}
$$

This optimization problem is non-convex. After we make some approximations, it is clear that the objective is equivalent to maximizing $\sum_{k=1}^{K}\left|h_{k}\right|^{2} p_{k}$. Thus, the problem (11) can be transformed into

$$
\max _{p_{k}} \sum_{k=1}^{K}\left|h_{k}\right|^{2} p_{k}
$$




$$
\begin{array}{ll} 
& \sum_{k=1}^{K}\left|\tilde{g}_{m}+\Delta_{m}\right|^{2} p_{k} \leq p_{m, t h} \\
\text { s.t. } & \sum_{k=1}^{K} p_{k} \leq P_{T} \\
& \operatorname{SIN} R_{k} \geq \gamma_{k, t h} \\
& \left|\Delta_{m}\right|^{2} \leq c_{m} \varepsilon_{m}^{2}
\end{array}
$$

Now we show that the above problem can be reformulated as a SOCP problem, following similar steps in [14]. Defining $\hat{\Delta}_{m}=\frac{1}{\sqrt{c_{m}}} \Delta_{m}$, the interference constraint in (3) is equivalent to

$$
\sum_{k=1}^{K} \max _{\left|\hat{\Delta}_{m}\right| \leq \varepsilon_{m}}\left|\tilde{g}_{m}+\hat{\Delta}_{m}\right| \sqrt{p_{k}} \leq \sqrt{p_{m, t h}}
$$

Using the triangle inequality and the Cauchy-Schwarz inequality with $\left|\hat{\Delta}_{m}\right| \leq \varepsilon_{m}$, it follows that

$$
\begin{aligned}
\sum_{k=1}^{K} \mid \tilde{g}_{m} \sqrt{p_{k}}+\hat{\Delta}_{m} \sqrt{c_{m}} & \sqrt{p_{k}} \mid \leq \sum_{k=1}^{K}\left(\left|\tilde{g}_{m} \sqrt{p_{k}}\right|+\left|\hat{\Delta}_{m} \sqrt{c_{m}} \sqrt{p_{k}}\right|\right) \\
& \leq \sum_{k=1}^{K}\left|\tilde{g}_{m} \sqrt{p_{k}}\right|+\left|\hat{\Delta}_{m} \sqrt{c_{m}} \sqrt{p_{k}}\right| \\
& \leq \sum_{k=1}^{K}\left|\tilde{g}_{m} \sqrt{p_{k}}\right|+\varepsilon_{m}\left|\sqrt{c_{m} p_{k}}\right|
\end{aligned}
$$

where the equality is achieved when

$$
\hat{\Delta}_{m}=\varepsilon_{m} \frac{\sqrt{c_{m} p_{k}}}{\left|\sqrt{c_{m} p_{k}}\right|}
$$

This indicates

$$
\sum_{k=1}^{K} \max _{\left|\hat{s}_{m}\right| \leq \varepsilon_{m}}\left|\left(\tilde{g}_{m}+\hat{\Delta}_{m}\right) \sqrt{p_{k}}\right|=\sum_{k=1}^{K}\left|\tilde{g}_{m} \sqrt{p_{k}}\right|+\varepsilon_{m}\left|\sqrt{c_{m} p_{k}}\right|
$$

So (13) is equivalent to

$$
\sum_{k=1}^{K}\left|\tilde{g}_{m}\right| \sqrt{p_{k}}+\varepsilon_{m}\left|\sqrt{c_{m}}\right| \sqrt{p_{k}} \leq \sqrt{p_{m, t h}}
$$

By combining (12) and (17), the optimization problem can be converted into a SOCP problem, as

$$
\max _{p_{k}} \sum_{k=1}^{K}\left|h_{k}\right|^{2} p_{k}
$$




$$
\begin{aligned}
& \sum_{k=1}^{K}\left|\tilde{g}_{m}\right| \sqrt{p_{k}}+\varepsilon_{m}\left|\sqrt{c_{m}}\right| \sqrt{p_{k}} \leq \sqrt{p_{m, t h}} \\
& \text { s.t. } \sum_{k=1}^{K} p_{k} \leq P_{T} \\
& S I N R_{k} \geq \gamma_{k, t h} \\
& \left|\Delta_{m}\right|^{2} \leq c_{m} \varepsilon_{m}^{2}
\end{aligned}
$$

Proposition 1: The power allocation problem (18) is a convex optimization problem with respect to $p_{k}$.

Proof: According to [15], the objective function in (18) is a convex function with respect to $p_{k}$. The left part of the first constraint in (18) is a convex function with respect to $p_{k}$. The second constraint is also convex. The interference generated by PUs together with the noise at $S U_{k}$ is $\sum_{j=1}^{M}\left|\hat{g}_{k}\right|^{2} p_{m}+\sigma_{k}^{2}=1$. Then, the third constraint can be transformed to

$$
\frac{\sum_{i=1, i \neq k}^{K}\left|h_{k}\right|^{2} p_{i}+\sum_{j=1}^{M}\left|\hat{g}_{k}\right|^{2} p_{m}+\sigma_{k}^{2}}{\left|h_{k}\right|^{2} p_{k}}=\frac{1}{\left|h_{k} / g_{m}\right|^{2} p_{k}} \leq \frac{1}{\gamma_{k, t h}}
$$

The left part of the inequality (19) is a nonnegative function, which is convex with respect to $p_{k}$. Since the objective function and the constraints are all convex, the problem (18) is a convex optimization problem. This completes the proof.

This optimization problem is very difficult to solve. So we will resort to numerical simulations to illustrate the convergence property of the sum utility of SUs.

\section{B. Analysis of NE}

Similar to [10], the best response of each SU is a dominant strategy (Generally, if the feasible strategy for a player is a dominant strategy, regardless of the strategies of the other players, its strategy will maximize the payoff function of the players). This implies that the above robust game has a unique NE.

\section{Numerical Results}

In this section, simulations are conducted to examine the performance of the proposed algorithm under flat fading channels. Moreover, we show the convergence property of power control under imperfect CSI. Assume that there are four SUs and four PUs in the cognitive network, i.e., $K=4$ and $M=4$. We choose $N_{t}=4$, the maximum transmit power for each $\mathrm{SU}$ is $p_{\max }=5 \mathrm{~W}$, the maximum interference level at $P U_{m}$ is $p_{m, t h}=10 \mathrm{~W}$, noise power as $\sigma^{2}=3 e^{-3} \mathrm{~W}$, PU transmission power as $p_{1}=\cdots=p_{M}=0.1 \mathrm{~W}$, the interference threshold as $I_{t h}=10 \mathrm{~dB}$, and the target SINR value for each SU is $\gamma_{1, t h}=\cdots=\gamma_{K, t h}=5 \mathrm{~dB}$, respectively. 


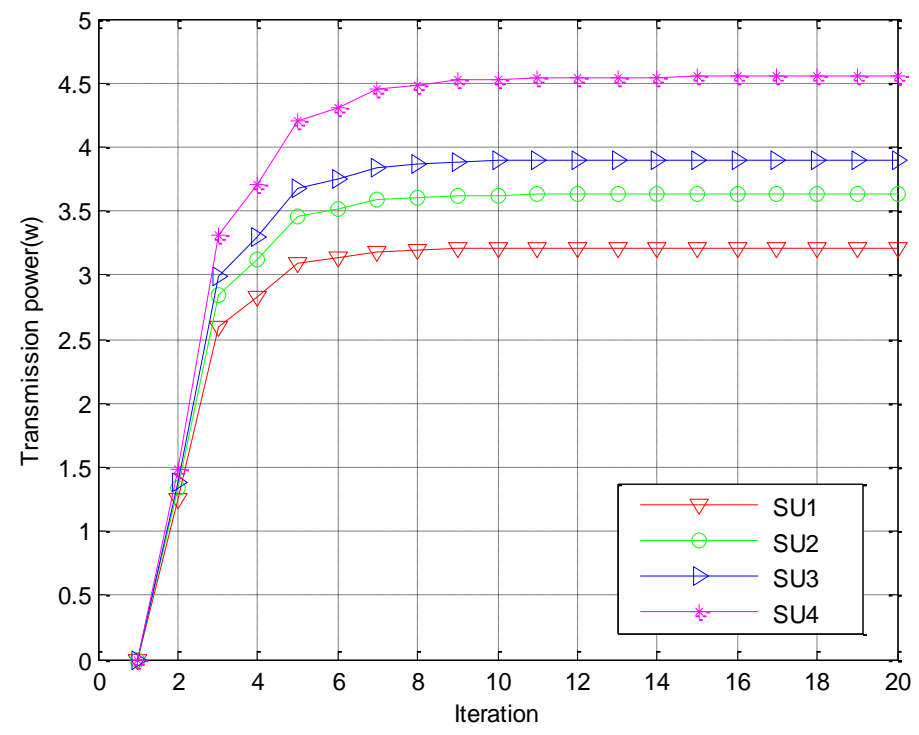

Figure 2. Convergence of Transmission Power for each SU

Figure 2 shows the convergence of transmission power for each SU, where the power initialization for each $\mathrm{SU}$ is the same as 0 . It is observed that the transmission power converges in 8 10 iterations due to the preceding update.

In the following, we show the impact of channel uncertainty on sum utility of SUs by comparing the imperfect CSI case with the perfect CSI case. According to the ratio of the channel coefficient from the SBS to a SU and the one from the SBS to the PU, i.e. $h_{k} / g_{m}$, the transmission power is allocated to each SU in a descending order. The sum utility obtained by a SOCP approximation algorithm [12] is evaluated. The estimation error is set to $\varepsilon_{m}^{2}=\left|g_{m}\right|^{2}$.

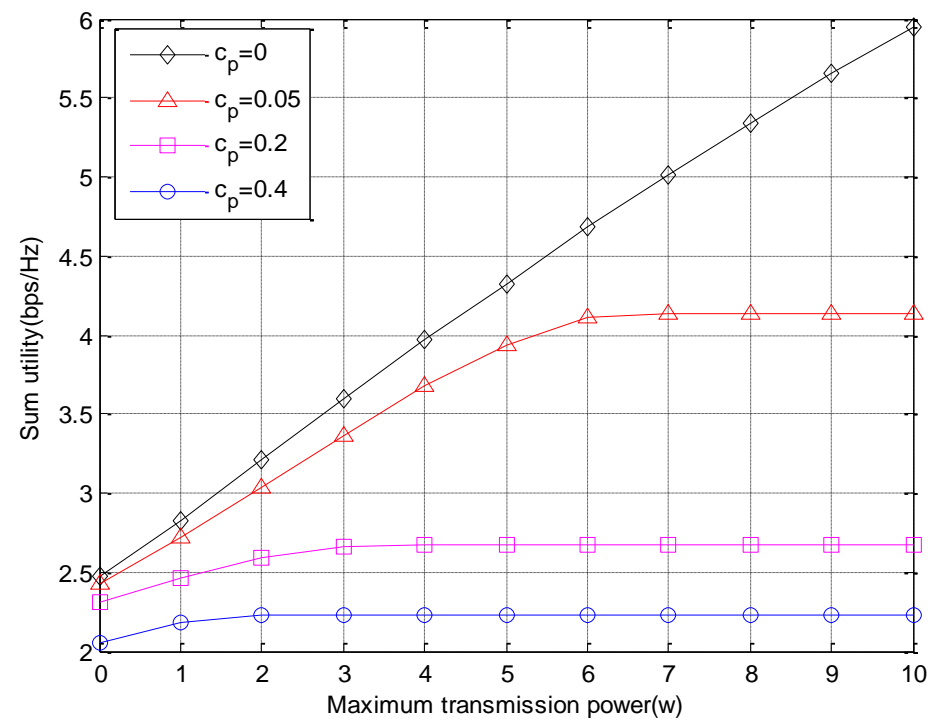

Figure 3. Sum Utility of SUs under Imperfect CSI 
Figure 3 shows the sum utility of SUs for a scenario with four SUs and four PUs. The uncertainty parameter is set to $c_{m}=0,0.05,0.2,0.4$, respectively. When $c_{m}=0$, the CSI is perfect (i.e., all the channel knowledge is perfectly known in the network). In addition, the noise power and the interference threshold between SUs and PUs are set to 1. Due to the CSI uncertainty, the sum utility is lower than that under perfect CSI. As the value of the uncertainty parameter increases, the sum utility becomes less. The sum utility of SUs under imperfect CSI converges with the increase of transmission power constraint of SUs.

\section{Conclusion}

In this paper, the robust power control problem for a CR network under imperfect CSI has been studied via game theory. An ellipsoid model was adopted to describe the CSI uncertainty. The problem was formulated as a robust game. The uncertainty optimization problem was transformed into a SOCP problem, in an effort to maximize the sum utility of SUs. Due to the CSI uncertainty, the sum utility is lower than that under perfect CSI. Simulation results showed the convergence property of the sum utility of SUs.

\section{Acknowledgements}

This research was supported by National Natural Science Foundation and Civil Aviation Administration of China (61071104).

\section{References}

[1]. S. Haykin, "Cognitive radio: Brain-empowered wireless communications", IEEE Journal on Selected Areas in Communications, vol. 23, no. 2, (2005), pp. 201-220.

[2]. J. Pang, G. Scutari, D. P. Palomar and F. Facchinei, "Design of cognitive radio systems under temperatureinterference constraints: A variational inequality approach”, IEEE Transactions on Signal Processing, vol. 58, no. 6, (2010), pp. 3251-3271.

[3]. A. He, S. Srikanteswara, K. K. Bae, T. R. Newman, H. Reed and W. H. Tranter, "Power consumption minimization for MIMO systems a cognitive radio approach", IEEE Journal on Selected Areas in Communications, vol. 29, no. 2, (2011), pp. 469-479.

[4]. L. Zheng and C. W. Tan, "Cognitive radio network duality and algorithms for utility maximization", IEEE Journal on Selected Areas in Communications, vol. 31, no. 3, (2013), pp. 500-5013.

[5]. C. Yang, J. Li, and Z, Tian, "Optimal power control for cognitive radio networks under coupled interference constraints: A cooperative game theoretic perspective”, IEEE Transaction on Vehicular Technology, vol. 59, no. 4, (2010), pp. 1696-1706.

[6]. X. Chen, Z. Zhao, H. Zhang and T. Chen, "Reinforcement learning enhanced iterative power allocation in stochastic cognitive wireless mesh networks", Wireless Personal Communications, vol. 57, no. 1, (2012), pp. 89-104.

[7]. P. Zhou, Y. Chang and J. A. Copeland, "Reinforcement learning for repeated power control game in cognitive radio networks", IEEE Journal on Selected Areas in Communications, vol. 30, no.1, (2012). pp. 5469.

[8]. K. Son, B. C. Jung, S. Chong and D. K. Sung, "Opportunistic underlay transmission in multi-carrier cognitive radio systems". In Proceedings of IEEE, wireless communications and networking conference, (2009), pp. 16.

[9]. M. Yang and D. Grace, "Cognitive radio with reinforcement learning applied to multicast downlink transmission with power adjustment”, Wireless Personal Communications, vol. 57, no. 1, (2011), pp. 73-87.

[10].F. Zhao, B. Li, H.-B. Chen and X. Z. Lv, "Joint beamforming and power allocation for cognitive MIMO systems under imperfect CSI based on game theory", Wireless Personal Communications, vol. 73, no. 3, (2013), pp. 679-694.

[11].A. J. G. Anandkumar, A. Anandkumar, S. Lambotharan and J. A.Chambers, "Robust rate-maximization game under bounded channel uncertainty", IEEE Transactions on Vehicular Technology, vol. 60, no. 9, (2011), pp. 4471-4486. 
[12].S. Parsaeefard, A. R. Sharafat, and M. van der Schaar, "Robust equilibria in additively coupled games in communications networks", IEEE GLOBECOM, (2011).

[13].Y. Xu and X. Zhao, "Robust Power Control for Multiuser Underlay Cognitive Radio Networks Under QoS Constraints and Interference Temperature Constraints", Wireless Personal Communications, vol. 75, (2014), pp. 2383-2397.

[14].J. Wang, G. Scutari, and D. P. Palomar, "Robust MIMO cognitive radio via game theory", IEEE Transactions on Signal Processing, vol. 59, no. 3, (2011), pp. 1183-1201.

[15].S. Boyd and L. Vandenberghe, Convex optimization. Cambridge University Press, (2004).

\section{Authors}

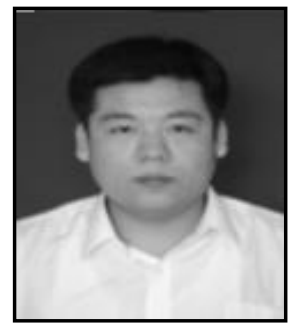

Guanglong Yang, he received the B.S. and the M.S. degrees from Northeast Agricultural University, Harbin, China, in 2003 and 2006, respectively. He is currently working toward the Ph.D. degree with the School of Electronics and Information Engineering, Harbin Institute of Technology. From 2007 to 2012, he was with Qiaohang Communication Company, Harbin, where he worked on the research and development of the digital trunking system. His research interests include power control and spectrum allocation for cognitive radio networks.

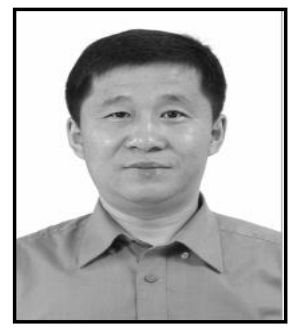

Xiao Wang, he received the B.S. degrees from Xi'an Jiao Tong University, Xianin, China, in 1988, received the M.S. degrees from Harbin Institute of Technology, Harbin, China, in 2005. He worked for Department of Electronics and Communication Engineering HIT as an associate professor and master's tutor since 2002. Now he is member of Heilongjiang Electronics Engineering Society. Ass Prof. Wang's research areas include the trunked mobile communication system, broadband multimedia mobile communication system, wireless network and digital transmission technology and other aspects of the scientific research and teaching work.

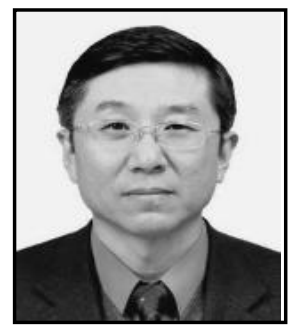

Xuezhi Tan, he received the B.S., M.S., and Ph.D. degrees from Harbin Institute of Technology, Harbin, China, in 1982, 1986, and 2005, respectively. From October 1988 to March 1990, he was a Visiting Scholar with Kyoto University, Kyoto, Japan. He is currently a Professor with the School of Electronics and Information Engineering, Harbin Institute of Technology. He is a Senior Member of the Chinese Institute of Electronics and the Chinese Institute of Communication. He had published more than 70 papers in international journals. His research interests include wireless communications, digital trunking communication, and cognitive radio. 